\title{
Verzeichnis der Briefe
}

852. Von Christian Andreas Biel, 4. Jan. $1764 \ldots \ldots \ldots 1$

853. An Christian Andreas Biel, Anfang Jan. 1764 .......... 1

854. Von Christiane Caroline Lucius, 10. Jan. 1764 . . . . . . . . . 2

855. An Christiane Caroline Lucius, 14. Jan. 1764 .......... 3

856. Von Christiane Caroline Lucius, 22. Jan. 1764 . . . . . . . . 5

857. An Friedrich Eberhard von Rochow, Jan. 1764 ......... 9

858. Von Christiane Caroline Lucius, 22. Febr. 1764 . . . . . . . . 9

859. An Christiane Caroline Lucius, 27. Febr. 1764 . . . . . . . . . 12

860. An Johanna Erdmuth von Schönfeld, 1. März 1764 . . . . . . 13

861. Von Johann Adolf Schlegel, 1. März 1764 . . . . . . . . . . . 14

862. An David Albin Hunger, 5. März 1764 . . . . . . . . . . . 15

863. Von Christiane Caroline Lucius, 6. März 1764 . . . . . . . . . . . 17

864. An Johann Ludwig Harscher, 13. März 1764 . . . . . . . . . . . 18

865. An Johann Adolf Schlegel, 18. März 1764 . . . . . . . . . . . 19

866. Von Christiane Caroline Lucius, 23. März 1764 . . . . . . . . 20

867. An Joachim Gottsche von Moltke, 24. März 1764 . . . . . . . 21

868. An Henriette Erdmuthe von Dieskau, 29. März 1764 . . . . . . 22

869. An Christiane Caroline Lucius, 31. März 1764 . . . . . . . . . . 22

870. An Christian Friedrich Pfeffel, 4. April 1764 . . . . . . . . . 23

871. Von Christiane Caroline Lucius, 8. April 1764 . . . . . . . . . . 24

872. An Johann Friedrich Voigt, 13. April 1764 . . . . . . . . . . . . 27

873. An Johann Georg Sulzer, 13. April 1764 . . . . . . . . . . . . 28

874. Von Johann Jacob Mack, 14. April 1764 . . . . . . . . . . . 28

875. An Johann Hartwig Ernst von Bernstorff, Mitte April 1764 . . 31

876. An Christiane Caroline Lucius, 17. April 1764 . . . . . . . . . 31

877. Von Christiane Caroline Lucius, 26. April 1764 . . . . . . . . 32

878. Von Johann Adolf Schlegel, 30. April 1764 . . . . . . . . . 33

879. Von Christian Andreas Biel, 1. Mai 1764 ... . . . . . . . . . 35

880. Von Johann Friedrich Wilhelm Jerusalem, Anfang Mai 1764 . . 36

881. Von Moritz August von Thümmel, 6. Mai 1764 . . . . . . . . . . 37

882. Von Christiane Caroline Lucius, 23. Mai 1764 . . . . . . . . . . . 37

883. An Jacob Friedemann von Werther, 28. Mai 1764 . . . . . . . . 40

884. An Johann Friedrich Wilhelm Jerusalem, 2. Juni 1764 . . . . . . 41

885. An Eléazar de Mauvillon, 2. Juni 1764 . . . . . . . . . . . 41

886. An Johanna Wilhelmine Biehle, 4. Juni 1764 . . . . . . . . . . 42

887. An Christiane Caroline Lucius, 5. Juni 1764 . . . . . . . . . . . . 43

888. Von Christiane Caroline Lucius, 11. Juni 1764 . . . . . . . . . . 43

889. An Erdmuthe Dorothea Magdalena Vitzthum von Eckstädt, 4. Juli 1764 . . . . . . . . . . . . . . . . . . . . 44

890. Von Christiane Caroline Lucius, 30. Juli 1764 . . . . . . . . . . . 44 
891. An Christiane Caroline Lucius, 8. Aug. 1764 . . . . . . . . . . 46

892. An Joachim Gottsche von Moltke, 9. Aug. 1764 . . . . . . . . . 55

893. An Friedrich Eberhard von Rochow, 10. Aug. 1764 . . . . . . . 57

894. An Ludwig August von Bötticher, 12. Aug. 1764 . . . . . . . . . 57

895. An Johanna Erdmuth von Schönfeld, 16. Aug. 1764 . . . . . 58

896. An Johanna Erdmuth von Schönfeld, 31. Aug. 1764 . . . . . . 60

897. Von Christiane Caroline Lucius, 31. Aug. 1764 . . . . . . . . 60

898. An Moritz Wilhelm Schlesier, 1. Sept. 1764 . . . . . . . . 67

899. An Christian Carl Persch, 4. Sept. 1764 . . . . . . . . . . 67

900. An Ludwig August von Bötticher, 6. Sept. 1764 . . . . . . . . . 68

901. An Moritz August von Thümmel, 10. Sept. 1764 . . . . . . . 68

902. An Christiane Caroline Lucius, 18. Sept. 1764 . . . . . . . . . . 69

903. Von Christiane Caroline Lucius, 21. Sept. 1764 . . . . . . . . 70

904. An Moritz Wilhelm Schlesier, 25. Sept. 1764 . . . . . . . . 73

905. An Johanna Wilhelmine Bieble, 4. Okt. 1764 . . . . . . . . . 74

906. An Johanna Erdmuth von Schönfeld, 18. Okt. 1764 . . . . . 74

907. An Johanna Erdmuth von Schönfeld, 20. Okt. 1764 . . . . . 74

908. Von Caroline Juliane Helene Kirchbof, 21. Okt. 1764 . . . . . 75

909. An Johanna Erdmuth von Schönfeld, 25. Okt. 1764 . . . . . 76

910. An Ernst Theodor Langer, 1. Nov. 1764 . . . . . . . . . . . 76

911. An Christiane Caroline Lucius, 1. Nov. 1764 . . . . . . . . . . . 77

912. Von Christiane Caroline Lucius, 14. Nov. 1764 . . . . . . . . 77

913. An Christiane Caroline Lucius, 21. Nov. 1764 . . . . . . . . . 80

914. Von Christiane Caroline Lucius, 28. Nov. 1764 . . . . . . . 82

915. An Johanna Erdmuth von Schönfeld, 30. Nov. 1764 . . . . . 83

916. An Christiane Caroline Lucius, 4. Dez. 1764 . . . . . . . . . . . 84

917. An Johanna Wilhelmine Bieble, 7. Dez. 1764 . . . . . . . . . 85

918. Von Christiane Caroline Lucius, 7. Dez. 1764 . . . . . . . . . 86

919. An einen ungenannten Pastor, $1764 \ldots \ldots . \ldots . \ldots 87$

920. An Herrn L., $1764 \ldots \ldots$. . . . . . . . . . . . . . 88

921. An Henriette Erdmuthe von Dieskau, 1764 . . . . . . . . . . . 88

922. An Ernst Haubold von Miltitz, 2. Jan. 1764 . . . . . . . . . 89

923. Von Christiane Caroline Lucius, 6. Jan. 1765 . . . . . . . . . 89

924. Von Christiane Caroline Lucius, 16. Jan. 1765 . . . . . . . . . 91

925. An Johanna Erdmuth von Schönfeld, 22. Jan. 1765 . . . . . . 95

926. An Wilhelm Friedrich Heinrich von Wartensleben, 22. Jan. 176595

927. An Johann Friedrich Wilhelm Jerusalem, 23. Jan. 1765 . . . . 96

928. An einen ungenannten Hauslehrer, 24. Jan. 1765 . . . . . . . 97

929. An Johanna Erdmuth von Schönfeld, 28. Jan. 1765 . . . . . . . 102

930. An Johanna Erdmuth von Schönfeld, 9. Febr. 1765 . . . . . . . 102

931. Von Johann Christoph Adelung, 13. März 1765 . . . . . . . . . 103

932. An Johann Adolf Schlegel, 15. März 1765 . . . . . . . . . . 104

933. An Johann Friedrich Wilhelm Jerusalem, 15. März 1765 . . . . 105

934. Von Christiane Caroline Lucius, 16. März 1765 . . . . . . . . 105

935. An Christiane Caroline Lucius, 21. März 1765 . . . . . . . . . . 107 
936. An Johann Adolf Schlegel, 26. März 1765 . . . . . . . . 108

937. Von Christiane Caroline Lucius, 1. April 1765 . . . . . . . . . 109

938. An Johanna Erdmuth von Schönfeld, 12. April 1765 . . . . . 111

939. Von Andreas Wagner, Mitte April 1765 . . . . . . . . . 112

940. An Gottlieb Leberecht Heyer, 25. April 1765 . . . . . . . . . 113

941. An Christiane Caroline Lucius, 4. Mai 1765 . . . . . . . . . 113

942. An Johann Adolf Schlegel, 6. Mai 1765 . . . . . . . . . . . 114

943. Von Christiane Caroline Lucius, 16. Mai 1765 . . . . . . . . 114

944. An Georg August von Breitenbauch, 20. Mai 1765 . . . . . 116

945. Von Christiane Caroline Lucius, 4. Juni 1765 . . . . . . . 116

946. An Christiane Caroline Lucius, 17. Juni 1765 . . . . . . . . 119

947. Von Christiane Caroline Lucius, 4. Juli 1765 . . . . . . . . . . 119

948. An einen ungenannten Sekretär, 20. Juli $1765 \ldots \ldots$. . . . 123

949. An Johanna Wilhelmine Biehle, 21. Juli 1765 . . . . . . . . 123

950. Von Christiane Caroline Lucius, 25. Juli 1765 . . . . . . . 123

951. An Joachim Gottsche von Moltke, 27. Juli 1765 . . . . . . . . 125

952. An Johanna Erdmuth von Schönfeld, 2. Aug. 1765 . . . . . 125

953. An Johanna Erdmuth von Schönfeld, 23. Aug. 1765 . . . . 126

954. An Jacob Hoogen, 25. Aug. 1765 . . . . . . . . . . . . . 127

955. An Moritz Wilhelm Schlesier, 15. Sept. 1765 . . . . . . . 128

956. Von Christiane Caroline Lucius, 18. Sept. 1765 . . . . . . 128

957. An einen ungenannten Baron, 19. Sept. $1765 \ldots \ldots \ldots$. . . . 130

958. An Johann Adolf Schlegel, 21. Sept. 1765 . . . . . . . . . 130

959. An Christian Carl Persch, 21. Sept. 1765 . . . . . . . 131

960. An Christiane Caroline Lucius, 22. Sept. 1765 . . . . . . . 131

961. Von Martin Friedrich Sörgel, 22. Sept. 1765 . . . . . . . . 132

962. An Johanna Erdmuth von Schönfeld, 1. Okt. $1765 \ldots \ldots . . .133$

963. Von Christiane Caroline Lucius, 12. Okt. 1765 . . . . . . 134

964. An Joachim Gottsche von Moltke, 16. Okt. 1765 . . . . . . 136

965. An Christian Felix Weiße, 25. Okt. 1765 . . . . . . . . 136

966. An Just Heinrich von Bötticher, 7. Nov. 1765 . . . . . . . . . 137

967. An Carl Friedrich Hindenburg, 25. Nov. 1765 . . . . . . . 137

968. An Carl Wilhelm Christian von Craussen, 1. Dez. 1765 . . . 138

969. An Joachim Gottsche von Moltke, 6. Dez. 1765 . . . . . . . . 139

970. An Johanna Wilhelmine Biehle, 11. Dez. 1765 . . . . . . . . 140

971. An Johann Adolf Schlegel, 24. Dez. 1765 . . . . . . . . . 141

972. Von Christiane Caroline Lucius, 30. Dez. 1765 . . . . . . . 141

973. An Friedrich Gottlieb Klopstock, 31. Dez. 1765 . . . . . . . . 143

974. An Johann Caspar Lavater, 31. Dez. 1765 . . . . . . . . . 144

975. Von Johann Nicolaus Meinhard, 31. Dez. 1765 . . . . . . . 144

976. An eine Ungenannte, $1765 \ldots \ldots \ldots \ldots$

977. An Pastor B***, $1765 \ldots \ldots \ldots \ldots$

978. An Christiane Caroline Lucius, 3. Jan. 1766 . . . . . . . . . 147

979. An Johanna Erdmuth von Schönfeld, 4. Jan. 1766 . . . . . . 147

980. Von Christiane Caroline Lucius, 20. Jan. 1766 . . . . . . . . 149 
981. An Joachim Gottsche von Moltke, 1. Febr. 1766 . . . . . . . . 150

982. An Johanna Erdmuth von Schönfeld, 18. Febr. 1766 . . . . . . 151

983. An Ernst Samuel Jacob Borchward, 18. Febr. 1766 . . . . . . . 152

984. Von Christian Ludwig von Hagedorn, 24. Febr. 1766 . . . . . . 153

985. An Christian Ludwig von Hagedorn, Febr. 1766 . . . . . . . . . 153

986. An Friedrich Eberhard von Rochow, 1. März 1766 . . . . . . . 154

987. Von Christiane Caroline und Friederike Auguste Lucius, 15. März

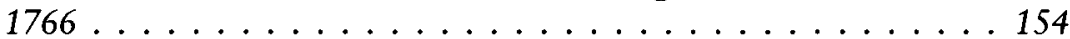

988. An Friedrich Eberhard von Rochow, 19. März 1766 . . . . . . 158

989. An Adam Gottlob von Moltke, 19. März 1766 . . . . . . . . . 159

990. An Friedrich Adolf von Kalkreuth, 19. März 1766 . . . . . . . 159

991. An Christiane Caroline Lucius, 20. März 1766 . . . . . . . . . . 160

992. An Ehrenfried Friedrich Biedermann, 22. März 1766 . . . . . . 161

993. Von Christiane Caroline Lucius, 25. März 1766 . . . . . . . . 162

994. An Johanna Erdmuth von Schönfeld, 29. März 1766 . . . . . . 164

995. An Christian Gottlob Heyne, 31. März 1766 . . . . . . . . . . 164

996. Von Christiane Caroline Lucius, 5. April 1766 . . . . . . . . . 165

997. An Johanna Erdmuth von Schönfeld, 8. April 1766 . . . . . . . 166

998. An Johanna Wilhelmine Biehle, 9. April 1766 . . . . . . . . 167

999. An Christiane Caroline Lucius, 13. April 1766 . . . . . . . . . . 167

1000. An Peter von Hohenthal, 27. April 1766 . . . . . . . . . 168

1001. Von Christoph Wilhelm von Kessel, 30. April 1766 . . . . . . 168

1002. An Andreas Wagner, 4. Mai 1766 . . . . . . . . . . . . . . . 169

1003. Von Jacob Bennelle, 8. Mai 1766 . . . . . . . . . . . . . 169

1004. Von Christian Gottlob Heyne, 11. Mai 1766 . . . . . . . . . . 170

1005. Von Christiane Caroline Lucius, 19. Mai 1766 . . . . . . . . . . 171

1006. Von Christiane Caroline Lucius, 24. Mai 1766 . . . . . . . . . 173

1007. An Johanna Wilhelmine Bieble, 9. Juni 1766 . . . . . . . . 173

1008. An Hans Gotthelf von Globig, 21. Juni 1766 . . . . . . . . . 174

1009. An Christiane Caroline Lucius, 23. Juni 1766 . . . . . . . . 175

1010. An Johann Daniel Reichel, 1. Juli 1766 . . . . . . . . . . 175

1011. An Friedrich von der Trenck, 8. Juli 1766 . . . . . . . . . . 176

1012. An Johann Carl Friedrich Hellfried, 18. Juli 1766 . . . . . . . . 177

1013. An Henriette Erdmuthe von Dieskau, 18. Juli 1766 . . . . . . . 179

1014. Von Christiane Caroline Lucius, 23. Juli 1766 . . . . . . . . 180

1015. An Johann Julius Christian Fulda, 4. Aug. 1766 . . . . . . . 182

1015. An Joachim Gottsche von Moltke, 10. Aug. 1766 . . . . . . . . 183

1017. An Friedrich Eberhard von Rochow, 12. Aug. 1766 . . . . . . . 184

1018. An Andreas Wagner, 13. Aug. 1766 ............... 184

1019. An Henriette Erdmuthe von Dieskau, 14. Aug. 1766 . . . . . . 185

1020. Von Christiane Caroline Lucius, 25. Aug. 1766 . . . . . . . 185

1021. An Carl Christian Gärtner, 28. Aug. 1766 . . . . . . . . . 187

1022. An Johanna Wilhelmine Biehle, 29. Aug. 1766 . . . . . . . . 188

1023. Von Joseph von Sonnenfels, 1. Sept. 1766 . . . . . . . . . . 188

1024. An Christiane Caroline Lucius, 9. Sept. 1766 . . . . . . . . . . . 189 
1025. An Christian Friedrich von Blankenburg, 12. Sept. 1766 . . . 189

1026. Von Abraham Gotthelf Kästner, 13. Sept. 1766 . . . . . . . 190

1027. An Carl Christian Gärtner, 17. Sept. 1766 . . . . . . . . . 191

1028. An Just Heinrich von Bötticher, 17. Sept. 1766 . . . . . . . 192

1029. An Andreas Wagner, 25. Sept. 1766 . . . . . . . . . 193

1030. An Ludwig August von Bötticher, 11. Okt. 1766 . . . . . . 193

1031. An Johann Adolf Schlegel, 23. Okt. 1766 . . . . . . . . . . 194

1032. An einen ungenannten Pastor, 18. November 1766 . . . . . 195

1033. An Christiane Caroline Lucius, 2. Dez. 1766 . . . . . . . . 195

1034. An Friedrich Eberhard von Rochow, 5. Dez. 1766 . . . . . . . 196

1035. Von Christiane Caroline Lucius, 5. Dez. 1766 . . . . . . . . 197

1036. An Christiane Caroline Lucius, 9. Dez. 1766 . . . . . . . . . 198

1037. An einen ungenannten Baron, 24. Dez. $1766 \ldots \ldots$. . . . . 198

1038. An Herrn von R., 1766 . . . . . . . . . . . . . . . 198

Zur Edition . . . . . . . . . . . . . . . . . . . 201

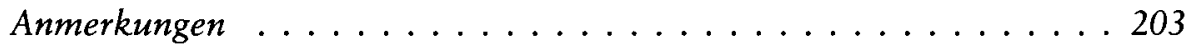

Zitierte Literatur . . . . . . . . . . . . . . . . . . . 291

Register . . . . . . . . . . . . . . . . . . . . . 293 
